\title{
Learning abstract relations from using categories
}

\author{
BRIAN H. ROSS and JUSTIN L. WARREN \\ University of Illinois, Urbana, Illinois
}

\begin{abstract}
When people learn categories, the importance of the features and relations in the category representation reflects both their diagnosticity for classification and their relevance to the use of the category. In earlier work in which the influence of category use on the representation has been shown, only cases in which the features and relations were simple, observable, and very specific were examined. Learners may begin to understand the underlying similarities of category members by using the categories. In the four experiments presented here, learners applied a simple category-specific formula to category members. The test results showed that the learners had incorporated relations among features from this use, including cases in which the relations were abstract. This learning occurred even though the relations were actually not predictive of category membership but just perceived to be so as a function of the use.
\end{abstract}

Categories are critical for intelligent thought and action. People can classify a large variety of objects, problems, and situations into categories and then can use knowledge of these categories to predict, explain, solve problems, and so forth. Although much work on classification has involved investigationinto how the observed perceptual features may be used for classification (e.g., see Kruschke, 1992; Lamberts, 1994; Medin \& Schaffer, 1978, Nosofsky, Palmeri, \& McKinley, 1994; Reed, 1972; Rosch, 1978), in many cases category members have deeper underlying similarities that make them cohere as a category (e.g., Murphy \& Medin, 1985). In the present research, we examine whether this coherence among members may arise from use of the category members to accomplish goals, such as problem solving. We begin by considering the underlying relations among the members of some categories and then examine how people may learn about categories during their goal-based use of category members.

\section{Coherence of Categories}

Although category members often may share many observable features, this perceptual similarity often reflects deeper underlying commonalities (Murphy \& Medin, 1985). These underlying commonalities provide a rationale for the category and allow us to include some members who may look rather atypical. As an example of natural categories, the category of mammals includes many objects that are quite perceptually similar, such as rats and mice, but also ones that look quite different, such as whales. In formal domains, expert problem solvers understand that even with clear similarities in

This work was supported by Grant NSF SBR 97-20304 from the National Science Foundation. We thank Seth Chin-Parker, J. Paul Minda, Gregory L. Murphy, Andrew Wills, and two anonymous reviewers for comments on the manuscript. Correspondence concerning this article should be addressed to B. H. Ross, Beckman Institute, University of Illinois, 405 N. Mathews Ave., Urbana, IL 61801 (e-mail: bross@s.psych.uiuc.edu). content, the classification of a problem depends on the deeper structure (see, e.g., Chi, Feltovich, \& Glaser, 1981). The point is that as people learn a category, they are often also learning what less observable commonalities make all of the items members of the same category.

An important aspect of coherence in many categorylearning situations is that of relations among the observable features. That is, the person learns not just what the observable features are, but also how they are interrelated, with the underlying commonalities often being derived from these interrelations. One area of research in which the importance of these interrelations has been very clear is the work on the effects of prior knowledge on categories and concepts (e.g., Malt \& Smith, 1984; Murphy \& Allopenna, 1994; Spalding \& Murphy, 1999). For instance, Murphy and Allopenna showed that learners can build coherent categories from features by using prior knowledge to link them, such as the idea of glacier vehicles to see that the features "is white" and "is insulated" may be related. Rehder and Ross (2001) found that people can even learn what they call abstract coherent categories - that is, categories that have no feature overlap but that specify a system of relations for interconnecting the features of all the category members. These different research projects have shown the importance of understanding relations among the observable features for providing a deeper coherence to the category.

Although most previous work has focused on the learning of object categories, it is important to realize that many of the categories we know are not about objects but, rather, about problems, situations, people, and so forth. In many of these cases, determining the exact features that are necessary for classification requires quite a bit of interpretation and abstraction.

\section{Category Use and Abstraction}

A critical question in category learning is how people learn which of the many features and relations of an item are important for category-related tasks. The research on 
classification in the last 25 years has shown how classification learning can lead to learning diagnostic featuresthat is, those features important for distinguishing to which category an item belongs (see Medin \& Smith, 1984, Ross \& Spalding, 1994, and Smith \& Medin, 1981, for reviews). However, the learning of categories involves more than learning to assign an item to the appropriate category.

Learning new categories involves both learning to classify and learning to use the categories to accomplish some goal. For example, as one is learning a new category of math problems, one is also often learning to apply the relevant formula to solve problems of that type. So, rather than learning by classification alone, as in the bulk of category-learning research, people often learn by classifying items into categories and then applying some category-related knowledge to the items. The importance of the features for later classification is not just related to their category predictiveness; rather, their importance is a joint function of their diagnosticity for classification and their relevance for category use (Ross, 1997). This effect on the weight of features has been shown across a variety of conditions and dependent measures (Ross, 1997, Experiments 1-5), when the use has been taught after the classification has been learned (Ross, 2000) and with young children (Ross, Gelman, \& Rosengren, 2002). Not only can using the category lead to a change in the weighting of features, it can also lead to selecting certain relations as being important within the category representation. For example, in problem-solving tasks, such as the decoding paradigm in the present experiments, learners incorporate the relations that are relevant for solving the problem into their category representations and use those relations for later classifications (Ross, 1997, Experiments 6 and 7, 1999).

A variety of recent research on category learning has emphasized how learners go beyond the simple features and relations that can be observed in the item. People often need to learn new features when learning new categories (e.g., Schyns, Goldstone, \& Thibaut, 1998; Schyns $\&$ Rodet, 1996), or they need to integrate perceptual information (Barsalou, 1999). Prior knowledge is often used in a variety of ways to help them to learn categories (Murphy \& Medin, 1985; see Heit, 1997, for overview). In addition, people often learn not just the simple features and relations, but also more abstract properties of the items, both when prior knowledge is used (e.g., Wisniewski \& Medin, 1994) and when complex categories are involved (e.g., Chi et al., 1981).

The abstractness of the properties is important across a variety of category types and category-related tasks. Although items have specific features, the category representations may include more abstract features and relations. Classification often requires abstraction from observable features. For example, classifying a data set as one that can be analyzed with a two-way analysis of variance (ANOVA) requires understanding how the particulars of the data set relate to the more abstract prop- erties of the ANOVA. Much of what we know and use about more complex categories includes abstract knowledge. For instance, consider the abstractness of the knowledge we have about what makes a person kind and how we would predict that person's behavior in a new situation. As another example, the knowledge we have about many math problem categories, such as permutations, is quite abstract; we can classify many differentlooking problems as being category members and apply that knowledge to solve all these different problems.

How are these abstract relations learned? It is possible that some underlying commonalities can be learned by comparing items classified in the same way (e.g., Spalding \& Ross, 1994). However, there is also evidence that classification may not be a good way to learn more abstract properties of the category or interrelations among the features (Medin, Dewey, \& Murphy, 1983; Yamauchi \& Markman, 1998, 2000b). Classification focuses the learners on the diagnostic features and relations, so learning about nondiagnostic properties that are important for using the category becomes less likely. In addition, learning requires a comparison across categories (to decide on the appropriate classification), so the difficulty of within-category comparison may be great (Yamauchi \& Markman, 2000a, 2000b).

Earlier investigations of the effects of category use have focused on learning specific features and relations, but there is some evidence that suggests that use of the categories might help people to learn features and relations that are more abstract. First, Yamauchi and Markman (2000b) have shown that learners who use categories to make inferences can more easily incorporate different manifestations of the same feature than can classification learners. That is, if a particular feature, such as roundheaded, was instantiated with a number of different types of round heads, inference learners had much less trouble learning the category than did classification learners, as compared with cases in which all the round heads were exactly the same. Second, when learning equation categories, those learners who solved the equations were more likely to see the underlying mathematical structure of the categories (Ross, 1996, 1997, Experiment 6).

The goal of the present experiments was to investigate whether the use of categories might provide an opportunity for learning about more abstract relations. Learners may begin to understand these relations from seeing the role of the relations in the use of the category. For example, suppose people are learning the principle of permutations for determining the number of different orderings of a subset of items. They may have learned to classify permutation problems by some keywords, but as they apply the formula to many diverse problems, they may begin to see the underlying commonality of how the orderings occur. This knowledge might then influence the classification of new problems, even if the keywords were missing, and provide a deeper understanding of this type of problem. 
Table 1

Sample Materials for Experiment 1

Decoding Formula

Spy A: $2 \mathrm{nd}+(3 \mathrm{rd}-6 \mathrm{th})+1 \mathrm{st}+5$ th

Spy B: 6 th $+(2 \mathrm{nd} / 5$ th $)+4$ th +3 rd

Sample Study Coded Messages

Spy A: D P 181842

Spy B: F S 745426

Sample Test Coded Messages

Spy A: --331212

Spy B: - -387643

Note-Ordinal numbers refer to the position of the number in the formula

\section{Overview of the Present Experiments}

In the present experiments, we addressed the question of whether the use of categories might lead to learning abstract relations by using a spy message decoding paradigm (Ross, 1997, Experiment 7, 1999). Subjects are given codes consisting of two letters and six numbers, such as FS342526, and they have to classify the messages as having come from Spy A or Spy B, using the letters to make this decision. After this classification, the subjects are asked to "decode" the message, by applying a spy-specific formula to the numbers in the message, as is shown in Table 1. The earlier work with this paradigm showed that learners incorporated the number relations relevant for decoding each spy's messages into their category representation (e.g., that the Spy B decoding always had the quotient of the second and fifth numbers equaling 2). The critical aspect of the manipulation was that these spy-relevant number relations were true for all of the coded messages. That is, the number relation important for decoding Spy B's messages, $2 \mathrm{nd} / 5 \mathrm{th}=2$, was also true for the Spy A messages (and similarly for the number relation relevant in Spy A messages). Although these relations were actually not predictive of category membership during learning (since they appeared in all the study messages of both spies), the subjects came to view them as category predictive because of the category use.

What is not clear from these earlier studies is how general the relations were that were learned about the category members. The number pairs used in each relation were the same at study and test. For example, some of the study items would have the second and fifth numbers be 6 and 3, respectively, and some of the test items would too. In addition, the relations were very specific, such as the quotient's equaling 2 , for all items. Learners could have classified the test messages on the basis of the presence of these specific number pairs or by noticing a simple relation in the message.

The goal of the present research was to investigate whether the learning from category use is more general in that it involves more than the specific number pairs and relations. In particular, can use of the category lead to a more abstract representation of the relations among the numbers in category members? If so, and if this re- lation influences subsequent classification, this research would provide evidence of a more abstract coherence among category members that arose from use of the category. This evidence would be especially strong because these abstract relations will be true of all items at studythat is, the members of both categories. Nonetheless, we would expect that each relation would be noticed only as a function of use in one category.

To clarify the issue, in the experiments, we addressed two related questions concerning the number pairs and the abstractness of the relations between the numbers. First, we examined whether the subjects were learning the numerical relations, and not just the exact number pairs from the study items (Experiment 1, replicated in Experiments 2-4). In later experiments, we also addressed the difference between old and new pairs (Experiments 3-4). Second, we investigated the abstractness of the relation by having number relations that were not specific numbers but that specified a general result for the relation, such as subtraction of one number from the other, leading to a negative number (Experiments 2-4). Does using the category-in this case, to decode the messageslead learners to incorporate general information about the number relations related to the use?

\section{EXPERIMENT 1}

In the first experiment, we tested whether the subjects would learn the relations among the numbers and incorporate them into their category representations or whether they would learn the specific number pairs. As in the earlier code experiments, there were two number relations, one relevant for each spy category (one relation from previous work, 2 nd $/ 5$ th $=2$, and a new one, 3 rd -6 th $=$ $-1)$. Instead of including all possible number pairs at study and test, we included only half at study, and then included all at test. The old pairs, the ones seen at study, allowed us to ensure that the earlier results would be replicated. The new pairs, the ones seen for the first time at test, allowed us to assess whether the learners had incorporated the relations into their category representations, or just the specific number pairs. As in all the earlier code experiments, all the study messages had both number relations, so the effect on subsequent classification was only a function of the category use.

\section{Method}

Subjects. The subjects were 16 University of Illinois students, who participated for class credit. The sessions lasted for 20-30 min.

Materials. Both the study and the test materials were code messages from fictional spies, Spy A and Spy B, as in Ross (1997, 1999). The study codes were two letters followed by six numbers. For half of the subjects, the letters PD or DP indicated Spy A, and the letters FS or SF indicated Spy B. For the other half of the subjects, the letter arrangement was switched, so that PD or DP indicated Spy B, whereas FS or SF indicated Spy A. The six-number sequence for all the messages had the following two constraints (see Table 1). First, the sixth number subtracted from the third was always equal to -1 . Second, the quotient of the second and the fifth numbers was always equal to 2 . There were eight sets of study items 
for each spy, divided into two sets of four. The study phase consisted of two blocks of eight codes.

There were 16 test items that contained dashed lines instead of letters. In every test item, only one of the critical number relations was present. Eight of these test items were old-pair items, which were the same pairs as those that had been in the study items in those positions. The other 8 items were new-pair items, which were numbers that had not occurred together as pairs in the critical positions during study.

All the study and test cards were printed labels on a $5 \times 7$ in. $(12.7 \times 17.8 \mathrm{~cm})$ index card.

Design. All the subjects received the same coded messages and made the same classif ications. In the study phase, every subject used one decoding formula for messages that began with PD or DP $[2 \mathrm{nd}+(3 \mathrm{rd}-6 \mathrm{th})+1 \mathrm{st}+5 \mathrm{th}]$ and a different formula for messages that began with FS or SF [6th $+(2 \mathrm{nd} / 5$ th $)+4$ th $+3 \mathrm{rd}]$. If the relations among the numbers were learned from using the decoding formulas and were incorporated into the category representations, classification test responses should be a function of the relations among the numbers (i.e., whether the 3 rd minus the 6 th was equal to -1 or the 2 nd divided by the 5 th was equal to 2 ), just as in previous work. Most important, if the subjects learned the relations, and not just the number pairs, the earlier finding should be true for the new pairs, as well as for the old pairs.

Procedure. All the subjects were told that they should think of themselves as being clerks in an intelligence-gathering operation. Their job was to determine which spy had sent the coded message and then apply the appropriate decoding formula so that the resulting number could be passed on to their supervisor for further decoding. They were told that the letters in the message could be used to determine which spy sent it. A pad of paper, a pencil, and the decoding formulas for each spy, which were typed on a piece of paper, remained in front of the subject during the study and the test phases.

For each study trial, the subject was handed a coded message, which he or she classified according to which spy sent it. Feedback was given, and then the subject applied the appropriate decoding formula, writing the parts and answers down on a piece of paper. Depending on the spy, the subjects had to apply one of the decoding formulas given in Table 1. For example, if the coded message was FS681342 for Spy B, the decoding formula [6th + (2nd/5th $)+$ 4 th +3 rd] would result in $(2+8 / 4+3+1=8)$. When he or she had finished, the subject informed the experimenter of his or her answer to the decoding formula. If it was incorrect, the experimenter asked the subject to apply the formula again. Once the subject had the correct answer, the experimenter reminded the subject of which spy it was and then presented the next item.

Before beginning the test phase, the experimenter informed the subject that all the messages had missing letters owing to garbled transmission. For each test trial, the subject was handed a coded message and had to determine which spy had sent it. After the classification of each trial, the subject made a confidence judgment for his or her answer on a scale from 1 (guessing) to 7 (certain).

Following the test trials, the subjects were asked a series of general questions to ascertain whether they had noticed the relations.

\section{Results and Discussion}

All statistical analyses used an $\alpha$ level of .05. Two dependent measures will be reported in all the experiments. First, we calculated the proportion of responses that were consistent with the use of the relation. Since each test message had only one relation instantiated in the same way as it had been at study (e.g., 2 nd $/ 5$ th $=2$ ), we counted the response as consistent if the message was assigned to the spy category for which this relation was part of the formula. Second, to obtain a more sensitive measure, we calculated the the subjects' overall mean confidence scores by summing the confidence judgments for each consistent answer, subtracting the confidence judgments for each inconsistent answer, and then dividing by the total number of items. A large positive confidence score (maximum of 7) indicated that the subjects had used the relation at test to classify consistently and were confident of their choices. A score of 0 meant that the relation had not been used, whereas a negative score indicated that the subjects were confident about choices that were inconsistent with the relation at test.

The design allowed us to ask and answer two questions. First, were the earlier results replicated? Yes, they were, as can be seen by the old-pair responses. The proportion consistent for old-pair items, in which the subjects had seen the same numbers in the study phase, was well above chance, with .91 of the classifications consistent with the relations $[t(15)=10.6]$. The mean confidence score for the old-pair items was 5.10 out of a possible mean of 7 , which was significantly different from a chance score of 0 , with $t(15)=8.33$. Thus, oldpair responses, as in previous experiments, showed that the subjects had learned the pairs or relations presented at study as a function of use and had used them to classify when the letters were not available.

Second, and of most interest, did the effect also occur with the new pairs, in which the particular number pairs had not been presented at study? Again, the answer was yes, with a proportion consistent of $.85[t(15)=9.13]$ and a mean confidence of $4.41[t(15)=7.07]$. These results suggest that the subjects do learn the relations involved in category use, and not just specific number pairs.

From the interviews with the subjects after the test trials, it was found that 12 subjects had noticed and were able to articulate both relations. Two subjects noticed only one relation, and 2 subjects did not notice either. It should be noted, however, that consistent classification could be performed when only one relation had been learned.

\section{EXPERIMENT 2}

The results of Experiment 1 indicate that learners incorporate information about a relation involved in category use into their category representation and that it can influence later classification. In Experiment 2, we investigated whether the relation could be more abstract and encompass a wider range of intermediate answers. Here, we changed the relations that would be calculated during decoding so that they would not have a single specific answer but, rather, a variety of answers that could be seen to be part of a larger set. In particular, the $2 \mathrm{nd} / 5$ th quotient equaled some number with 0.5 in it (i.e., 0.5 , $1.5,2.5,3.5,4.5)$ and the 3 rd -6 th difference now equaled some negative number. If learners can incorporate these more abstract relations into their category representations as a function of use, they should be able to classify later test items that include only one of these relations. As in previous experiments, all the study items had both of these relations satisfied. 
Table 2

Sample Materials for Experiments 2, 3, and 4

Decoding Formula

Spy A: $(3 r d-6$ th $)+5+1 s t+2 n d+5$ th

Spy B: $(2 \mathrm{nd} / 5$ th +1$) * 4$ th +6 th +3 rd

Sample Study Coded Messages

Spy A: D P 921847

Spy B: F S 652626

Sample Test Coded Messages

Spy A: - - 552215

Spy B: --543481

Note-Ordinal numbers refer to the position of the number in the formula.

\section{Method}

Subjects. The subjects were 16 University of Illinois students, who participated for pay. The sessions lasted for 30-45 min.

Materials. The study and the test materials had a few changes from Experiment 1. The same decoding formulas were used, except that the relations were made more abstract: The 3rd - 6th was always equal to a negative number and the 2 nd $/ 5$ th was always equal to a number that ended with a half (e.g., 3.5; see Table 2 for more examples). There were 12 sets of study items for each spy, divided into two sets of 6 . The study phase consisted of two blocks of 12 coded messages.

To avoid obvious differences in the decoding answers, two changes were made to the decoding formulas and messages. First, the decoding answers always were integers. The intermediate answer that included a half was multiplied by an even number. Second, we adjusted the formula and numbers so that the final answers for both formulas had similar ranges.

There were 20 test items, which, as in Experiment 1, possessed only one critical number relation. Twelve of the items were old-pair items, whereas the other 8 were new-pair items. The numbers used in each condition can be seen in Table 3 . The study and test cards were constructed like those in Experiment 1.

Design. As in Experiment 1, all the subjects received the same coded messages and made the same classifications. In Experiment 2, however, a counterbalancing variable was added, so that each of the decoding formulas was used by half the subjects for Spy A and by the other half for Spy B.

Procedure. The procedure was the same as that in Experiment 1, except that the decoding formulas for the two spies were given on two separate pieces of paper. During the study phase, after feedback was given, the experimenter placed the index card for that trial on the appropriate formula sheet for the correct spy. This change increased the salience of the connection between the spy and its decoding formula.

Some minor changes were added to ensure that the subjects learned the letters classified. First, the subjects were reminded twice in the instructions that the letters in the message could be used to determine which spy sent it. Second, a learning criterion was also added into the study phase so that the subjects must have classified 10 out of the 12 trials correctly after the first study block before being allowed to continue to the test phase.

\section{Results and Discussion}

All the subjects met the classification criterion of 10 of 12 correct in the study phase by the second block.

Again, the proportion of old-pair items classified consistently with the relation was $.84[t(15)=8.34]$, with a mean confidence score of $3.64[t(15)=7.61]$. This result indicates that the subjects probably were able to incorporate a more abstract relation into the category repre- sentation, although it is possible that they were responding on the basis of the particular number pairs.

A clear decision between these two possibilities can be obtained by looking at the new-pair items, because these number pairs had not occurred during study. These items also showed a strong influence of the relation on test performance. The proportion consistent for the newpair items was .77 $[t(15)=5.84]$, with a mean confidence score of $2.58[t(15)=4.91]$. This result demonstrated that the subjects had learned the abstract relation between the critical numbers.

From the final questions asked of the subjects, it was found that 11 subjects noticed both relations, 4 subjects noticed only one, and 1 subject did not notice either.

\section{EXPERIMENT 3}

The first two experiments indicated two ways in which learning from category use can lead to some abstraction in a representation. First, learners were able to consistently classify test items with new pairs of numbers, suggesting that they had learned the relation between the number pairs (Experiments 1 and 2). Second, learners were able to consistently classify items for which the relation value had not been seen before, indicating that they had learned a more general relation, such as one number's being larger than another (Experiment 2).

Experiment 3 was conducted for two purposes. First, it provided a replication of the learning of abstract relations. Because Experiment 2 was the first indication that people may learn more abstract relations from use, a replication was necessary. Second, in this experiment, we further examined what subjects learn about specific number pairs versus the relation. To better understand how category use influences a representation, it is important to understand what information is being incorporated. In particular, is there evidence that the subjects have incorporated any specific information about number pairs so that they are better able to classify old pairs than new pairs or to classify them more confidently? The earlier experiments were designed to examine transfer to the new pairs, so the old and new pairs were not coun-

Table 3

Critical Number Pairs Used in Experiment 2

\begin{tabular}{lll}
\hline 3rd -6 th & 2nd/5th \\
\hline \multicolumn{3}{c}{ Old Pairs } \\
$1-7$ & & $4 / 8$ \\
$2-6$ & $6 / 4$ \\
$4-5$ & $2 / 4$ \\
$3-5$ & & $9 / 6$ \\
$6-9$ & & $3 / 6$ \\
$3-8$ & & $5 / 2$ \\
& & \\
$2-5$ & & \\
$3-6$ & & $3 / 2$ \\
$2-9$ & & $9 / 2$ \\
$1-9$ & $7 / 2$ \\
\hline
\end{tabular}


Table 4

Old-Pair Items for Each Condition in Experiment 3

\begin{tabular}{ccccc}
\hline \multicolumn{2}{c}{ Condition A } & & \multicolumn{2}{c}{ Condition B } \\
\cline { 2 - 2 } 3rd -6th & 2nd/5th & & 3rd -6th & 2nd/5th \\
\hline $6-9$ & $4 / 8$ & & $5-8$ & $1 / 2$ \\
$1-8$ & $2 / 4$ & & $3-5$ & $3 / 6$ \\
$4-6$ & $3 / 2$ & & $1-7$ & $5 / 2$ \\
$1-2$ & $9 / 6$ & & $4-9$ & $6 / 4$ \\
$2-6$ & $7 / 2$ & & $2-3$ & $9 / 2$ \\
\hline
\end{tabular}

Note-The old-pair items for Condition A served as the new-pair items in Condition B, and vice versa.

terbalanced. Thus, how to interpret the small but consistent advantage for the old pairs in the first two experiments was unclear, but this difficulty was remedied in Experiment 3.

\section{Method}

Subjects. The subjects were 24 University of Illinois students, who participated for pay. The sessions lasted for 35-45 min.

Materials. The study and test materials were the same as those in Experiment 2, except that the particular number pairs in the old and new pairs were split into two sets for counterbalancing. Half of the subjects received one set of number pairs in the critical relations during study, whereas the other half of the subjects received the other set (see Table 4 for the number pairs used). There were 10 coded messages for each spy during study, divided into two sets of 5 . The study phase consisted of two blocks of 10 coded messages.

There were 20 test items, consisting of 10 old-pair items and 10 new-pair items. The new-pair items were the number pairs from the set not seen during study. The study and test cards were constructed like those in Experiment 2.

Design. The design was similar to that in Experiment 2, except for the counterbalancing of materials, as described in the Materials section.

Procedure. The procedure was exactly the same as that in Experiment 2, except that the learning criterion was changed so that the subjects needed to classify 8 out of 10 study items correctly after the first study block before proceeding to the test phase.

\section{Results and Discussion}

All the subjects met the classification criterion of 8 out 10 correct in the study phase by the second block.

Old-pair items replicated the effect of Experiment 2, with a proportion consistent of $.75[t(23)=5.64]$ and a mean confidence score of $2.48[t(23)=4.93]$. In addition, the results again show that the subjects learned the abstract relations and used them to classify. The newpair items were again responded to far above chance, with a proportion consistent of .71 $[t(23)=4.88]$ and a mean confidence score of $2.18[t(23)=4.53]$.

The new question in this experiment was whether the learners would incorporate anything about the specific number pairs into the category representation: Would there be a difference between old-pair and new-pair items? The .04 difference in proportion consistent $(.75$ versus .71) was not statistically significant $[t(23)=1.51]$, nor was the .3 difference in mean confidence [ 2.48 vs. $2.18 ; t(23)=1.20]$. Thus, there was no significant difference, although the old pairs showed a small numerical advantage.
From the final questions, it was found that 12 subjects noticed both constraints, 4 subjects noticed one constraint, and 8 subjects did not notice either constraint.

\section{EXPERIMENT 4}

The purpose of Experiment 3 was to replicate the learning of the abstract relations and to investigate whether there was any specificity effect for the particular number pairs. The results showed a clear replication for the learning of abstract relations, but the examination of the specificity effect was not as clear. The results indicated no significant difference, but the old pairs did lead to slightly more consistent classification. The main purpose of Experiment 4 was to investigate the old-pair versus new-pair contrast with a change, to emphasize any existing specificity effect. In particular, we constructed the materials in each set in a way that would minimize the overlap of intermediate products (quotients or differences) with the other set, so that any learning of specific old pairs would not help performance for the new pairs. The 3rd - 6th differences were unique for each of the eight pairs, but this could not be done with the 2 nd $/ 5$ th constraints, owing to the small set of quotients that could be created with number ranging from 1 to $9(0.5,1.5,2.5$, 3.5 , and 4.5$)$. Although we could not totally separate the intermediate products from the critical relations, the change did make the two material sets less similar and made it easier to see whether any real specificity effect existed. This experiment also provided one more replication of whether learners can incorporate an abstract relation from category use into a category representation.

\section{Method}

Subjects. The subjects were 24 University of Illinois students, who participated for pay. The sessions lasted for 35-45 min.

Materials. The study and test materials were the same as those in Experiment 3, except for changes in the particular number pairs to minimize overlap of intermediate products across the two sets (see Table 5 for the number pairs used). There were eight sets of study items for each spy, divided into two sets of four. The study phase consisted of two blocks of eight coded messages.

There were 16 test items, consisting of 8 old-pair items and 8 new-pair items. The study and test cards were constructed like those in Experiment 3.

Design. The design was the same as that in Experiment 3.

Procedure. The procedure was the same as that in Experiment 3, except that the learning criterion was changed so that the subjects needed to classify seven out of eight trials correctly after the first study block before being allowed to continue to the test phase.

\section{Results and Discussion}

All the subjects met the classification criterion of seven out of eight correct in the study phase by the second block.

As in previous experiments, there was an effect for the old-pair items with a proportion consistent of $.65[t(23)=$ $3.75]$ and a mean confidence score of $1.47[t(23)=3.62]$. The results again showed that the subjects can incorporate the abstract relation into the category representation 
Table 5

Old-Pair Items for Each Condition in Experiment 4

\begin{tabular}{ccccc}
\hline \multicolumn{2}{c}{ Condition A } & & \multicolumn{2}{c}{ Condition B } \\
\cline { 2 - 4 } 3rd -6th & 2nd/5th & & 3rd -6th & 2nd/5th \\
\hline $2-9$ & $3 / 2$ & & $2-4$ & $5 / 2$ \\
$3-7$ & $9 / 2$ & & $2-5$ & $7 / 2$ \\
$1-7$ & $3 / 6$ & & $3-8$ & $4 / 8$ \\
$5-6$ & $2 / 4$ & & $1-9$ & $1 / 2$ \\
\hline
\end{tabular}

Note-The old-pair items for Condition A served as the new-pair items in Condition B, and vice versa.

and use it to classify. The new-pair items demonstrated an effect as well, with a proportion consistent of .64 $[t(23)=2.66]$ and a mean confidence score of $1.07[t(23)=$ 2.18].

Most critically, no significant difference was found between the old pair and the new-pair items. The difference in proportions consistent was .01 $[t(23)=0.11]$, and the difference in mean confidence scores was $0.4[t(23)=$ 1.11]. Thus, even though we tried to minimize any overlap between the two sets to make a specificity effect easier to obtain, there was no significant difference between old-pair and new-pair items. However, all of the differences across the two experiments with counterbalanced items (Experiments 3 and 4) did show a small, nonsignificant advantage for the old-pair items, so some small specificity effect cannot be ruled out.

The final questions indicated that only 3 subjects noticed both relations, whereas 11 noticed one relation and 10 subjects did not notice either relation.

\section{GENERAL DISCUSSION}

These experiments show that the use of a category can have a profound effect on its mental representation even when the information learned is an abstract relation among the observable features. In addition, the change in the representation occurred solely as a function of the learners' perceptions of the relevance for use, since the relations were not actually predictive of category membership. Thus, the use of categories, outside of classification, can lead to a deeper and more abstract understanding of categories.

\section{Summary of Results}

Four major results were of interest in these experiments. The first, replicated in all four experiments, was that the use of the categories affected subsequent classification. These experiments demonstrated that a number relation learned through applying the category-specific decoding formula to category members can be incorporated into category representations and can affect later classification. The subjects incorporated the relations into the category representations even though they were not predictive of category membership.

The second result found in all four experiments was that the subjects learned the relations-not just the specific number pairs. In all cases, responses to new-pair test items were significantly above chance.
The third result, in Experiments 3 and 4, was that there was no significant difference between old-pair and newpair test items. This result suggests that the learners were not incorporating specific item information but, rather, information about the relation. However, this finding is qualified by the small nonsignificant advantages consistently found for the old-pair items, as will be discussed in the next section. At any rate, any old-new difference was small, relative to the overall effect of the relation.

The fourth and main result, found in Experiments 2, 3, and 4 , was that the subjects were able to incorporate more abstract relations into their category representations. The intermediate result did not have to be a specific number but could be a more abstract relation among the numbers. This is the first evidence of an abstract relation among the features being learned through the use of category knowledge.

\section{Specificity Effect}

Although this fourth result was the main goal of the paper, the second and third results bear further discussion. The new-pair test items were often classified consistently with the relation used for that category during the study phase. This finding suggests that the knowledge of the relation was not tied to specific number pairs seen at study. We cannot rule out the possibility that the relation was generated at test, but this seems unlikely, for two reasons. First, to do this, the subjects would have had to remember a number of the critical specific pairs from study and yet never have understood the commonality among these pairs until a test item was shown. Second, the confidence for the new pairs was nearly as high as that for the old pairs.

Two further analyses provide a fuller understanding of the difference between the old and the new pairs. First, the old pairs did have a small nonsignificant advantage. If one combines the results of Experiments 3 and 4 to have 48 subjects, the differences are still not significant but are closer, especially for the more sensitive confidence measure $[t(47)=0.91$ for proportions with a sign test split of 17 vs. $12 ; t(47)=1.62$ for confidence with a 26 vs. 18 split]. However, even if there was a small advantage for the old pairs, it was very small, relative to the overall relation effect.

Second, splitting the subjects in Experiments 3 and 4 by whether they noticed the relations (as determined by the questions at the end of the experiment) gives a more complete picture. For the 15 subjects who noticed both relations, performance was high on both the old and the new items (mean proportion of .84), and there was virtually no difference between the old and the new items (differences of .009 in proportions and .03 in confidence). However, for the 18 subjects who did not notice either relation, performance was not much above chance (.53), but there was a larger, although still nonsignificant, old/new effect $[.04$ in proportions, $t(17)=0.84 ; .53$ in confidence, $t(17)=1.30$ ]. (The 15 subjects who noticed one relation had intermediate overall performance and intermediate old-new differences.) Thus, it appears 
that if the subjects did not notice the relation, they showed a small specificity effect, perhaps owing to one or two number pairs. However, if the subjects did notice the relations, they showed almost no old/new difference.

Note, however, that the claim that abstract relations can be learned from using the category does not entail a claim that the specifics of the relations cannot also be incorporated into the category representation. If the materials allowed for more distinctive differences between the old and the new items, the specificity effect may well have been larger, even for the subjects noticing the relation. At any rate, the claim is not that people do not learn specific relations or pairs but, rather, that they may learn abstract relations as well. It may well be, as was seen here, that once learners understand the relation between features, the importance of the exact features may be less.

\section{Learning Abstract Relations From Use of Categories}

The main finding of these experiments is that the use of the categories (to decode the message) allowed the subjects to learn abstract relations. Remember that the exact same relations occurred for all the items in both categories, so the test results show that learning was a function of use. The evidence of abstract relation learning is a critical link in assessing the importance of category use for category-learning effects. Although much work on category learning has focused on learning observable features or simple relations among observable features, many categories do not have identical lower order relations (e.g., 3rd -6 th $=-1$ ) but, rather, have commonalities among category members at a more abstract or higher level (e.g., 3rd - 6th is a negative number).

To better understand how category use might influence learning, it is worth considering two fundamental issues in category learning. First, given the large number of features and relations in complex items, how do people learn which features and relations are important for classification and for other category-related goals? Second, given that category representations often include more abstract information about features and relations than can be simply observed in the items, how is this abstract information learned? We speculate here that various uses of categories, other than classification, may be critical for answering both of these questions.

First, category use may provide a heuristic about which features and relations are important. That is, given a large number of features and relations, people are more likely to pay attention to and incorporate into their category representations those features and relations that are involved in their use of the category. The work on classification has assumed this idea and provided strong evidence for it in terms of the classification, in that diagnostic features are attended to more than nondiagnostic features and serve as the bases for later classifications. The speculation here is a little broader in two ways. One, the benefit occurs not just for the classification function of categories, but for other category uses as well, such as inference and problem solving. Two, the features and relations attended to and incorporated into the category representations for a use of the category affect not just that use (which has been shown by the classification research) but, potentially, any other use of the category. There is evidence for this idea for both features and relations. The category use work with the symptom/disease paradigm showed that equally category-diagnostic features were responded to very differently at the end of classification and inference learning: Those features relevant to the inference were more likely to be correctly classified than were those irrelevant to the inference; in addition, they were generated earlier and viewed as having occurred more frequently (Ross, 1997). The research using the code paradigm showed that the relations involved in the use influenced later classifications even though they were not at all diagnostic (since they occurred equally in both categories). Thus, category use may provide one means of restricting which of the many features and relations are important for a category.

Second, abstraction may be easier with some category uses other than classification. As mentioned earlier, Yamauchi and Markman (2000b) found that if a feature was instantiated in multiple ways (e.g., different types of round heads), inference learning was much less affected than was classification learning. That is, the inference learners appeared to be able to learn the more abstract feature of round head (as compared with a particular round head) more easily than were the classification learners. One possible account for this difference is that inference learning (and many nonclassification uses of categories) focuses on a single category, rather than across categories (see Murphy \& Ross, 1994, for evidence in inference tasks). For example, when decoding a Spy A message, the learner knows this is a Spy A message and can think about how it compares with other Spy A messages, rather than with all the other messages. When classifying an item, the learners must consider multiple categories to decide what category the item should be assigned to, making abstraction within a category more difficult. Thus, category uses other than classification may help learners to develop more abstract features and relations.

\section{Generality of the Code Paradigm}

These results were found with one paradigm, and further research will need to address their generality across other paradigms. However, it is important to note that the present experiments had a number of factors that might have made learning abstract relations more difficult than it would be in many situations. First, the use of the category required only a simple, fast application of an arithmetic formula, so there was little opportunity or reason for reflecting upon any deep commonalities of the category. Second, the use required no learning, just a simple application. In fact, it is not clear how the learning of the abstract relations (Experiments 2, 3, and 4) would have helped in even speeding up the application; knowing that a quotient ends in 0.5 does not tell one what the quotient 
is. Third, the features involved in the classification and the use were separate, and the subjects were told explicitly that the letters could be used to classify but that the decoding formulas would be applied to the numbers.

In many situations, none of these factors would be true, and we believe that their absence might lead to a larger influence of category use. Many complex problemsolving situations force learners to think more about the reasons for their category membership, both to help in solving later problems and because learners often assume that there is an underlying commonality to these situations, whereas the subjects in experiments may not. If category use required some learning, people might be forced to try to understand how it is being similarly applied to the different category members. Finally, if the features and relations involved in the classification and use were overlapping (e.g., part of the treatment of a disease involves testing the diagnostic symptoms), the use might have a greater effect on what is incorporated into the category representation and how central that information is considered for the category.

\section{Conclusions}

Categories are used for a variety of functions, and the representation must be able to be used to accomplish all these functions (Solomon, Medin, \& Lynch, 1999). Classification is essential; the person needs to know what type of object, situation, or problem the current one is. However, once that is known (or even once a likely candidate is determined; Murphy \& Ross, 1994), the representation of the category has to include information that will allow the person to accomplish his or her goal. As one is learning, knowledge relevant to the classification and knowledge relevant to the uses needs to be incorporated into the category representation. The learning of the use may provide one means by which abstract features and relations may be incorporated to help learners understand the underlying commonalities of category members.

\section{REFERENCES}

Barsalou, L. W. (1999). Perceptual symbol systems. Behavioral \& Brain Sciences, 22, 577-660.

Chi, M. T. H., Feltovich, P. J., \& Glaser, R. (1981). Categorization and representation of physics problems by experts and novices. $C o g$ nitive Science, 5, 121-152.

HEIT, E. (1997). Knowledge and concept learning. In K. Lamberts \& D. Shanks (Eds.), Knowledge, concepts, and categories (pp. 7-41). Cambridge, MA: MIT Press.

KRUSCHKE, J. K. (1992). ALCOVE: An exemplar-based connectionist model of category learning. Psychological Review, 99, 22-44.

LAMBERTs, K. (1994). Flexible tuning of similarity in exemplar-based categorization. Journal of Experimental Psychology: Learning, Memory, \& Cognition, 20, 1003-1021.

Malt, B. C., \& Smith, E. E. (1984). Correlated properties in natural categories. Journal of Verbal Learning \& Verbal Behavior, 23, 250269.

Medin, D. L., Dewey, G. I., \& Murphy, T. D. (1983). Relationships between item and category learning: Evidence that abstraction is not automatic. Journal of Experimental Psychology: Learning, Memory, \& Cognition, 9, 607-625.
Medin, D. L., \& Schaffer, M. M. (1978). Context theory of classification learning. Psychological Review, 85, 207-238.

Medin, D. L., \& S Mith, E. E. (1984). Concepts and concept formation. Annual Review of Psychology, 35, 113-138.

Murphy, G. L., \& Allopenna, P. D. (1994). The locus of knowledge effects in concept learning. Journal of Experimental Psychology: Learning, Memory, \& Cognition, 20, 904-919.

Murphy, G. L., \& Medin, D. L. (1985). The role of theories in conceptual coherence. Psychological Review, 92, 289-316.

MurPhy, G. L., \& Ross, B. H. (1994). Predictions from uncertain categorizations. Cognitive Psychology, 27, 148-193.

Nosofsky, R. M., Palmeri, T. J., \& McKinley, S. C. (1994). Rule-plusexception model of classification learning. Psychological Review, 101, 53-79.

REed, S. K. (1972). Pattern recognition and categorization. Cognitive Psychology, 3, 382-407.

REHDER, B., \& Ross, B. H. (2001). Abstract coherent categories. Journal of Experimental Psychology: Learning, Memory, \& Cognition, 27, 1261-1275.

Rosch, E. (1978). Principles of categorization. In E. Rosch \& B. Lloyd (Eds.), Cognition and categorization (pp. 27-48). Hillsdale, NJ: Erlbaum.

Ross, B. H. (1996). Category representations and the effects of interacting with instances. Journal of Experimental Psychology: Learning, Memory, \& Cognition, 22, 1249-1265.

Ross, B. H. (1997). The use of categories affects classification. Journal of Memory \& Language, 37, 240-267.

Ross, B. H. (1999). Postclassification category use: The effects of learning to use categories after learning to classify. Journal of Experimental Psychology: Learning, Memory, \& Cognition, 25, 743-757.

Ross, B. H. (2000). The effects of category use on learned categories. Memory \& Cognition, 28, 51-63.

Ross, B. H., Gelman, S. A., \& Rosengren, K. (2002). Children's category-based inferences affect classification. Manuscript submitted for publication.

Ross, B. H., \& Spalding, T. L. (1994). Concepts and categories. In R. Sternberg (Ed.), Handbook of perception and cognition: Vol. 12. Thinking and problem solving (pp. 119-148). San Diego: Academic Press.

Schyns, P. G., Goldstone, R. L., \& Thibaut, J.-P. (1998). The development of features in object concepts. Behavioral \& Brain Sciences, 21, 1-54.

Schyns, P. G., \& Rodet, L. (1996). Categorization creates functional features. Journal of Experimental Psychology: Learning, Memory, \& Cognition, 23, 681-696.

Smith, E. E., \& Medin, D. L. (1981). Categories and concepts. Cambridge, MA: Harvard University Press.

Solomon, K. O., Medin, D. L., \& Lynch, E. (1999). Concepts do more than categorize. Trends in Cognitive Sciences, 3, 99-104.

Spalding, T. L., \& Murphy, G. L. (1999). What is learned in knowledgerelated categories? Evidence from typicality and feature frequency judgments. Memory \& Cognition, 27, 856-867.

Spalding, T. L., \& Ross, B. H. (1994). Comparison-based learning: Effects of comparing instances during category learning. Journal of Experimental Psychology: Learning, Memory, \& Cognition, 20, 12511263.

WisNiEwsKi, E. J., \& MEdin, D. L. (1994). On the interaction of theory and data in concept learning. Cognitive Science, 18, 221-282.

Yamauchi, T., \& Markman, A. B. (1998). Category-learning by inference and classification. Journal of Memory \& Language, 39, 124148.

YAMAUCHI, T., \& MARKMAN, A. B. (2000a). Inference using categories. Journal of Experimental Psychology: Learning, Memory, \& Cognition, 26, 776-795.

Yamauchi, T., \& Markman, A. B. (2000b). Learning categories composed of varying instances: The effect of classification, inference, and structural alignment. Memory \& Cognition, 28, 64-78.

(Manuscript received June 15, 2001; revision accepted for publication February 15, 2002.) 